\title{
Investigation of interaction of $\mathrm{THz}$ radiation with blood components
}

\author{
$\underline{\text { S. I. Gusev }}{ }^{1}$, T. Zhang ${ }^{1}$, V. Yu. Soboleva ${ }^{1}$, Yu. A. Kononova ${ }^{2}$, V. A. Guseva ${ }^{1}$, \\ P. S. Demchenko ${ }^{1}$, E. A. Segykh ${ }^{1}$, M. K. Khodzitsky ${ }^{1}$ \\ ${ }^{1}$ ITMO University, St. Petersburg, Russia, mail@gusev-spb.ru \\ ${ }^{2}$ Federal Almazov North-West Medical Research Centre, St. Petersburg, Russia
}

Last decades $\mathrm{THz}$ radiation becomes more popular for spectroscopy and imaging tasks. One of the most important directions is biomedical diagnostics [1]. In comparing with visible, IR and microwave frequency ranges, $\mathrm{THz}$ radiation has more unexplored topics [2]. In context of wide spreading of diabetes mellitus disease, the blood glucose sensing in $\mathrm{THz}$ frequency range has especial importance.

This work represents list of our results, obtained step-by-step to develop non-invasive technique of blood glucose measurement. There is list of performed approaches described below: some show dependence between blood glucose concentration and optical properties, some experiments devoted to increasement of sensitivity to glucose and some to make measurement by non-invasive way.

All physical experiments had been performed with $\mathrm{THz}$ time-domain spectroscopy (TDS) in Terahertz Biomedicine lab of ITMO University.

First experiment objects were cotton clothes impregnated by fresh blood and measured in transmission way. [3] Each sample had been extracted from the same patient during short time period and had different glucose concentrations.

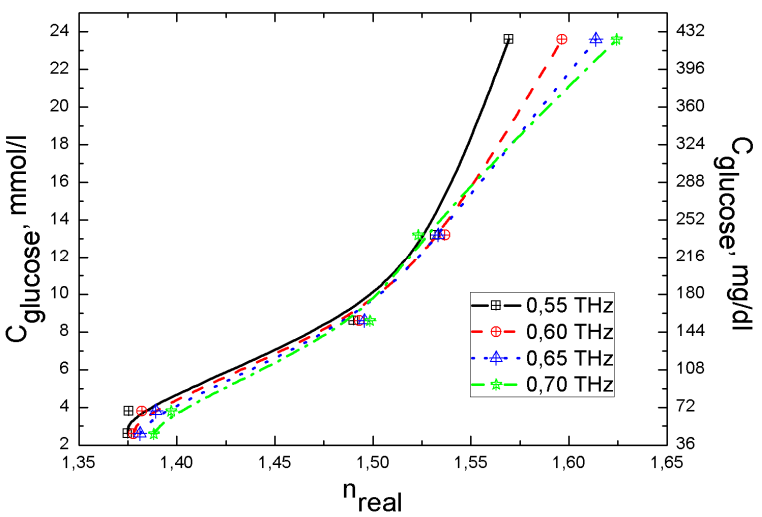

Fig. 1. Correlation between glucose concentration and refractive index had been observed at some frequencies

Second group of experiments was performed to reassure in results and increase accuracy. To prevent blood coagulation, water evaporation and provide stable thickness of the blood layer, special container had been produced. [4] It consisted from bottom and top parts, produced from polymethyl methacrylate. There were 2 experiments where blood layer was located inside the container. Blood samples had been from the same patient up to 8 times per experiment.
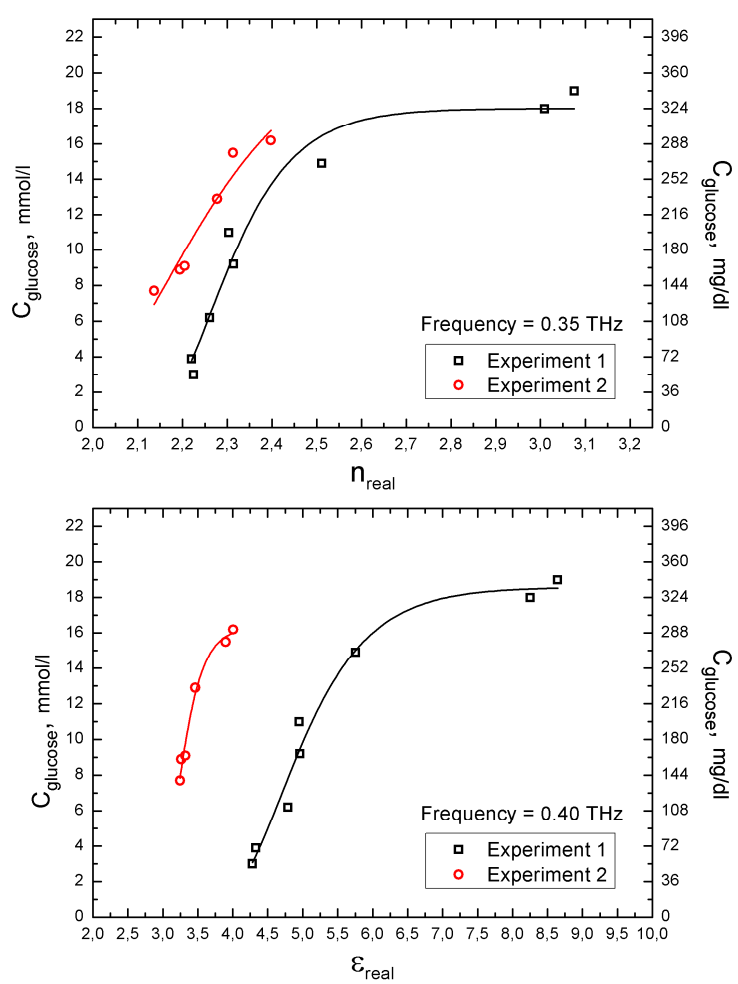

Fig. 2. Correlation behavior repeatability: for refractive index (top graph) as well as for permittivity (bottom graph)

Experimental results represented above showed method sensitivity to glucose level on condition of stable concentrations of other blood components (there were single patient during experiments). To estimate variability there were about 50 biosamples extracted from different patients, where concentration of components varies at each patient. [5] Each blood sample was analyzed for concentration of glucose, bilirubin, triglycerides, creatinine and uric acid by standard clinic laboratory tests. To observe influence of each component concentration on blood optical properties, observed parameters had been grouped by following principle: one parameter is varied, other are fixed.

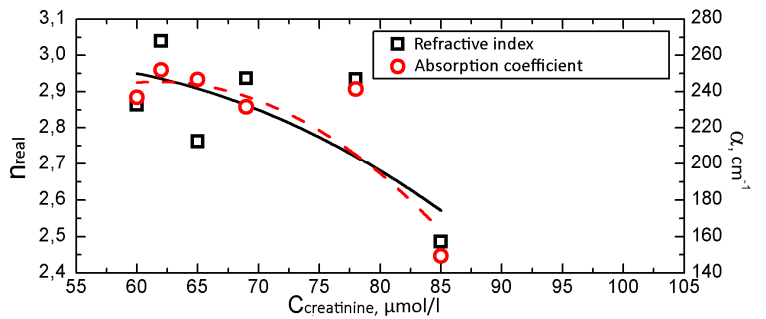



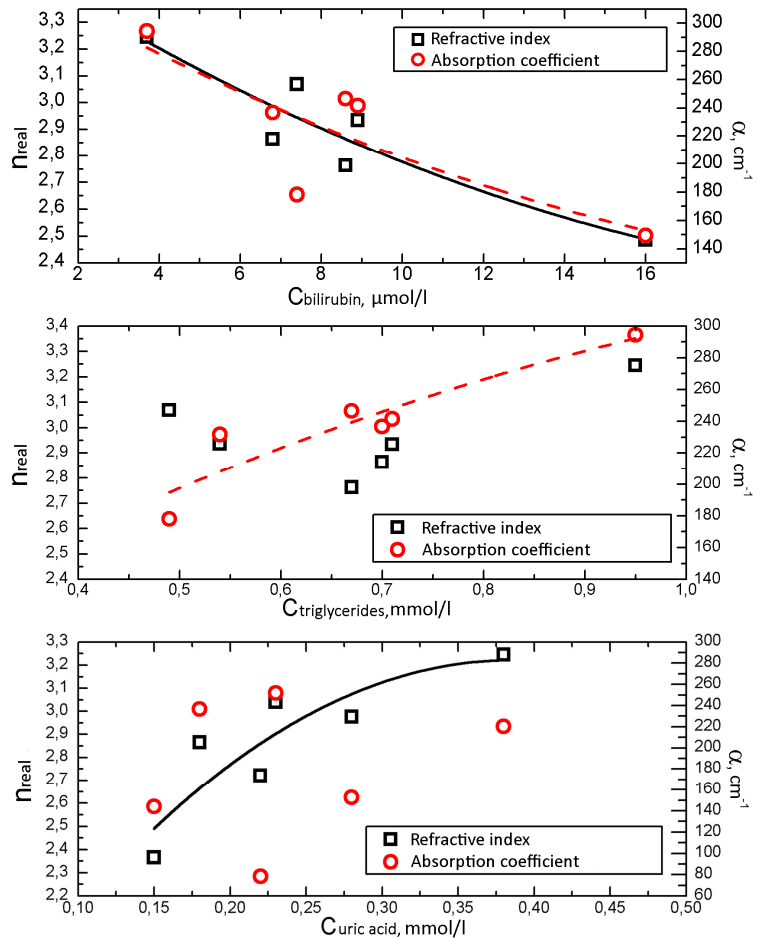

Fig. 3. In a number of cases there is possible to observe as blood optical properties in $\mathrm{THz}$ frequency range (such as refractive index and absorption coefficient) depend on concentrations of blood components.

To enhance sensitivity to glucose level there was performed simulation with band-pass aluminum metasurface with cross-shaped resonators sprayed on polyethylene terephthalate substrate. [6] Model consisted from this structure covered by thin layer of blood samples with the constant thickness. Each blood sample had different glucose concentration, their optical properties calculated before had been used during simuations.

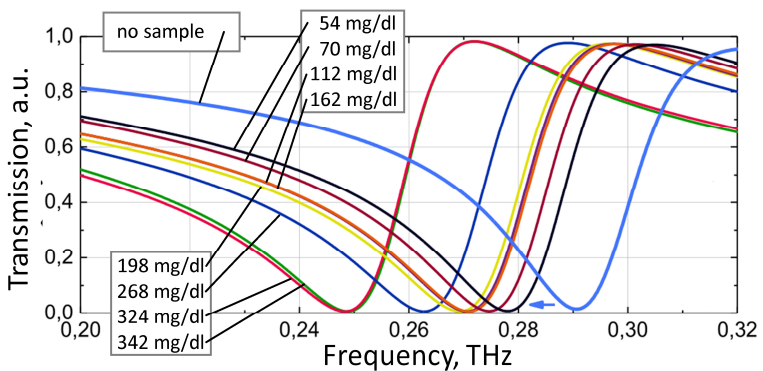

Fig. 4. With increasing of blood glucose concentration, we observe resonance frequency shift, which value directly correlates with blood glucose concentration.

For non-invasive technology development reasons, simulation of reflective experiment had been performed. [7] Described object consisted from nail plate and nail bed. Nail bed contains significant part of capillary filled by blood. Nail plate has stable thickness, flat surface and low absorption at $\mathrm{THz}$ frequencies. [8] As a result, modeled structure included listed objects, which were described by frequency dispersions of permittivity, calculated before with $\mathrm{THz}$ TDS in transmission mode.

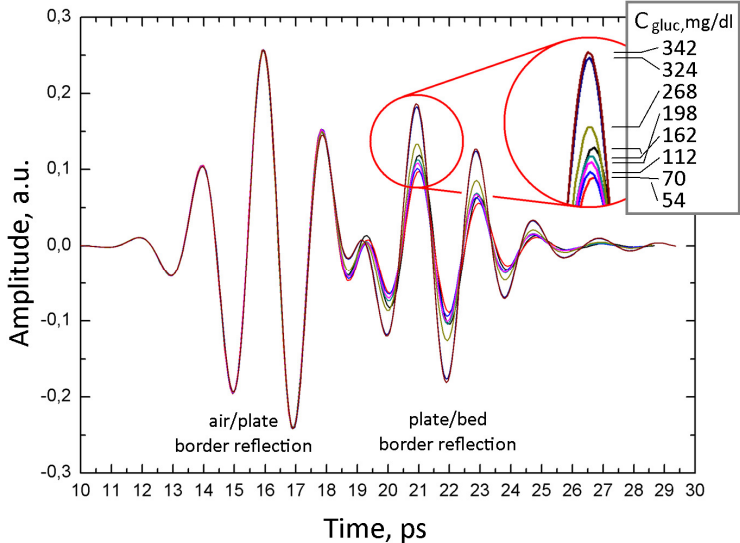

Fig. 5. Peak and peak-to-peak amplitude variations in reflection from plate/bed border shows tendency to correlation with blood glucose concentration.

Described series of studies shows dependence of blood glucose level and blood optical properties such as refractive index and permittivity. Influence of bilirubin, triglycerides, creatinine, uric acid concentration on whole blood optical properties had been investigated. There is metasurface-based method of increasing selectivity to glucose had been modeled and proposed. Non-invasive experiment using THz TDS had been simulated. Correlation of reflection amplitude and blood glucose had been observed.

There results showed $\mathrm{THz}$ radiation applicability and optimistic opportunities in the task of noninvasive glucose measurement.

\section{References}

1. Pickwell, E., Wallace, V. P. Biomedical applications of terahertz technology // Journal of Physics D: Applied Physics. 2006. V. 39, No. 17. P. R301.

2. Tonouchi, M. Cutting-edge terahertz technology // Nature photonics. 2007. V. 1, No. 2. P. 97.

3. Gusev, S. I., et al. Blood optical properties at various glucose level values in $\mathrm{THz}$ frequency range // Clinical and Biomedical Spectroscopy and Imaging IV. 2015. V. 9537. P. 95372A.

4. Gusev, S. I., et. al. Influence of Glucose Concentration on Blood Optical Properties in THz Frequency Range // Chinese Optics. 2018. V. 11, No. 2. P. 182-189.

5. Zhang, T., et. al. Qualitative analysis of blood components in terahertz frequency range // Scientific and Technical Journal of Information Technologies, Mechanics and Optics. 2018. V. 18, No. 5. (in Russian).

6. Soboleva, V. Yu., Gusev, S. I., Khodzitsky, M. K. Physical properties of macroscopically inhomogeneous media // Scientific and Technical Journal of Information Technologies, Mechanics and Optics. 2018. V. 18, No. 3. P. 377-383. (in Russian).

7. Gusev, S. I., et al. Study of glucose concentration influence on blood optical properties in $\mathrm{THz}$ frequency range // Nanosystems: Physics, Chemistry, Mathematics. 2018. V. 9, No. 3. P. 389-400

8. Guseva, V. A., et al. Optical properties of human nails in THz frequency range // Journal of Biomedical Photonics \& Engineering. 2016. V. 2, No. 4. 